\title{
Mathematical Model of Helical Gear Topography Measurements and Tooth Flank Errors Separation
}

\author{
Huiliang Wang, ${ }^{1}$ Xiaozhong Deng, ${ }^{2}$ Jianhai Han, ${ }^{2}$ Jubo Li, ${ }^{2}$ and Jianjun Yang ${ }^{2}$ \\ ${ }^{1}$ School of Mechanical Engineering, Northwestern Polytechnical University, Xian 710072, China \\ ${ }^{2}$ School of Mechatronics Engineering, Henan University of Science and Technology, Luoyang, Henan 471003, China \\ Correspondence should be addressed to Xiaozhong Deng; dxz01@163.com
}

Received 23 June 2015; Revised 7 November 2015; Accepted 29 November 2015

Academic Editor: Jean J. Loiseau

Copyright (c) 2015 Huiliang Wang et al. This is an open access article distributed under the Creative Commons Attribution License, which permits unrestricted use, distribution, and reproduction in any medium, provided the original work is properly cited.

\begin{abstract}
During large-size gear topological modification by form grinding, the helical gear tooth surface geometrical shape will be complex and it is difficult for the traditional scanning measurement to characterize the whole tooth surface. Therefore, in order to characterize the actual tooth surfaces, an on-machine topography measurement approach is proposed for topological modification helical gears on the five-axis $\mathrm{CNC}$ gear form grinding machine that can measure the modified gear tooth deviations on the machine immediately after grinding. Combined with gear form grinding kinematics principles, the mathematical model of topography measurements is established based on the polar coordinate method. The mathematical models include calculating trajectory of the centre of measuring probe, defining gear flanks by grid of points, and solving coordinate values of topology measurement. Finally, a numerical example of on-machine topography measurement is presented. By establishing the topography diagram and the contour map of tooth error, the tooth surface modification amount and the tooth flank errors are separated, respectively. Research results can serve as foundation for topological modification and tooth surface errors closed-loop feedback correction.
\end{abstract}

\section{Introduction}

Gear transmission is a major form of transmitting machine movement and power. In particular, cylindrical helical gear pair is widely used in the high speed and heavy load mechanical transmission because its transmission is stable and its carrying capacity is strong. Precision and quality have been the critical issues for manufacturing helical gears as the tooth surface accuracy directly affects the performance of equipment [1]. It is known that gear quality could be affected by various types of errors and factors, such as machine errors, heat treatment distortions, variation of grinding forces, and unpredictable factors. In practice, gear form grinding can be a very effective means for eliminating tooth flank errors of heavy-duty or large-size gear. On the other hand, tooth surface modification is another effective technology that can reduce the vibration and noise of gear transmission device, improve meshing state, effectively prevent the edge contact, and prolong the working life of gear [2-4].

There are two methods for gear measurement method: polar coordinate measuring method [5] and rectangular coordinate measuring method [6]. Zhang et al. [7] expressed the conversion formula between the polar angle and the generating angle during measuring tooth flank errors by the polar coordinate measuring method. Gao et al. $[8,9]$ proposed a new pretravel quantitative calibration method on the basis of analyzing the cause of the pretravel, which could improve the on-machine measurement accuracy using touch trigger probe. Nafi et al. [10, 11] studied an error separation method based on multistep redundancy method, in which the errors of the measurement machine tool, standard ball, probe, and probe tip producing in the measurement process are separated from calibration results by multistep measuring. Shih and Chen [12] proposed free-form flank correction in helical gear grinding using a five-axis computer numerical control gear profile grinding machine, but the errors of the actual tooth surface were assessed using the gear measurement centre, not on-machine measurement. Kobayashi et al. $[13,14]$ calculated the angle of the grinding wheel installation and optimized contact line between the grinding wheel and the work gear, in order to achieve the accuracy of the gear flank profile. Lee et al. $[15,16]$ fabricated modified cylindrical 
gear drive that could reproduce precisely the predesigned fourth-order polynomial function of transmission error.

The traditional tooth surface measurement is measured in the central line of profile and longitudinal direction, with reference to the standard involute helical surface modification as well as the error. Due to tooth surface for topological modification, this measure cannot satisfy the modification design and processing requirements. The traditional tooth surface measurement process is to measure the tooth flank errors when the profile error is in the central tooth height and the longitudinal error is in the central tooth width, respectively. By measuring, the topography measurement can obtain the modification amount as well as the error with reference to the standard involute helical surface. For the topological modification tooth surface, this measure process cannot satisfy the requirements of the modification design and machining. Therefore, how to implement topography measurement and dominate completely the tooth surface error information is the key points of this study for the topological modification tooth surface.

The outline of the remainder of the paper is as follows. In Section 2, an on-machine measurement model of helical gear based on gear form grinding is developed. A numerical example is the main subject of Section 3. In Section 4, the characterization of tooth surface error and modification amount are shown, respectively. Finally, some conclusions are drawn in Section 5.

\section{On-Machine Measurement}

2.1. Coordinate Systems of Measurement. Based on the topological modification surface equation as a theory equation, helical gear flanks are defined by a grid of points, where an elementary sketch affects the ideal tooth surface. According to the measuring probe along the tooth surface measurement path planning, the measured displacement is recorded by a data acquisition system. The topography diagram is drawn according to the tooth surface errors, which can observe clearly the error distribution of 45 points on the tooth surface and provide the theoretical basis of the tooth surface error feedback correction and closed-loop manufacture of helical gears.

The measurement of the helical gear is inspected along the radial workpiece using straight probe, which is shown in Figure 1. During machining and measuring the workpiece, whether the locating datum is consistent is the key. The premise of accurately measuring the tooth surface deviation on the machine is that the space position of the measuring coordinate system and the space position of the workpiece coordinate system coincide too. The origin of the measurement coordinate system $S_{C}$ is locating at point $C\left(x_{1 C}, y_{1 C}, z_{1 C}\right)$ which is the centre of rotary table. When gear grinding and measuring, it is located with datum end face and axis using rotary table and top, respectively. The theoretical tooth surface design coordinate system origin and workpiece coordinate system origin coincide as $O_{1}$. On-machine measurement can improve the accuracy of grinding tooth surface due to avoiding the reinstallation of the workpiece. The relative position of the space of the coordinate systems

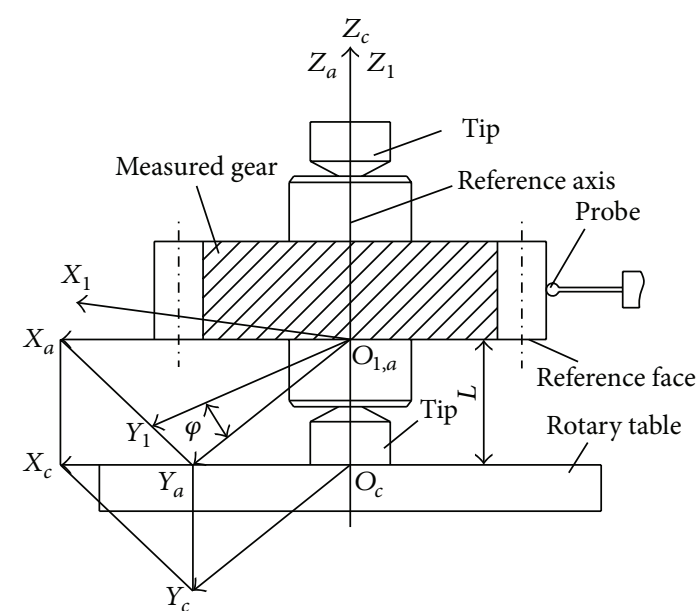

FIGURE 1: Coordinate systems of on-machine measurement.

is shown in Figure 1. $S_{1}\left(X_{1}, Y_{1}, Z_{1}\right)$ is workpiece coordinate system, $S_{c}\left(X_{c}, Y_{c}, Z_{c}\right)$ is measuring coordinate system, $L$ is the axial distance of the two coordinate system origins, and $\varphi$ is tooth surface rotation angle. The space position relation between coordinate system $S_{1}$ and coordinate system $S_{c}$ is determined by the transformation matrix $\mathbf{M}_{c 1}$.

The tooth flank form geometry can be generally represented by a position vector and a unit normal vector in the workpiece coordinate system $S_{1}$ as

$$
\begin{aligned}
& \mathbf{r}_{1}=\mathbf{r}_{1}\left(u_{1}, \theta_{1}\right), \\
& \mathbf{n}_{1}=\mathbf{n}_{1}\left(u_{1}, \theta_{1}\right) ;
\end{aligned}
$$

here, $\left(u_{1}, \theta_{1}\right)$ are generating surface parameters.

For the accurate detection of actual processing tooth surface, the theoretical tooth surface should be transformed to the gear measuring coordinate system $S_{C}$, by coordinate transformation which is obtained as follows:

$$
\begin{aligned}
& \mathbf{r}_{c}=\mathbf{M}_{c 1} \mathbf{r}_{1}\left(u_{1}, \theta_{1}\right), \\
& \mathbf{n}_{c}=\mathbf{M}_{c 1} \mathbf{n}_{1}\left(u_{1}, \theta_{1}\right) ;
\end{aligned}
$$

here,

$$
\mathbf{M}_{c 1}=\left[\begin{array}{cccc}
\cos \varphi & \sin \varphi & 0 & 0 \\
-\sin \varphi & \cos \varphi & 0 & 0 \\
0 & 0 & 1 & L \\
0 & 0 & 0 & 1
\end{array}\right]
$$

2.2. Mathematical Model of Measurement. The use of a polar coordinate to inspect gears is becoming increasingly useful in modern gear manufacturing, especially on gear form grinding. On-machine measurement of gear profile error is arranged based on the CNC gear form grinder, which has five digital servo closed-loop controlled axes: three rectilinear motions $(X, Y$, and $Z$ ) and two rotational motions $(A, C)$. Here, $C$ is the rotation axis of work gear, and $X$ is the radial motion for feeding the wheel down to tooth depth. The theory 


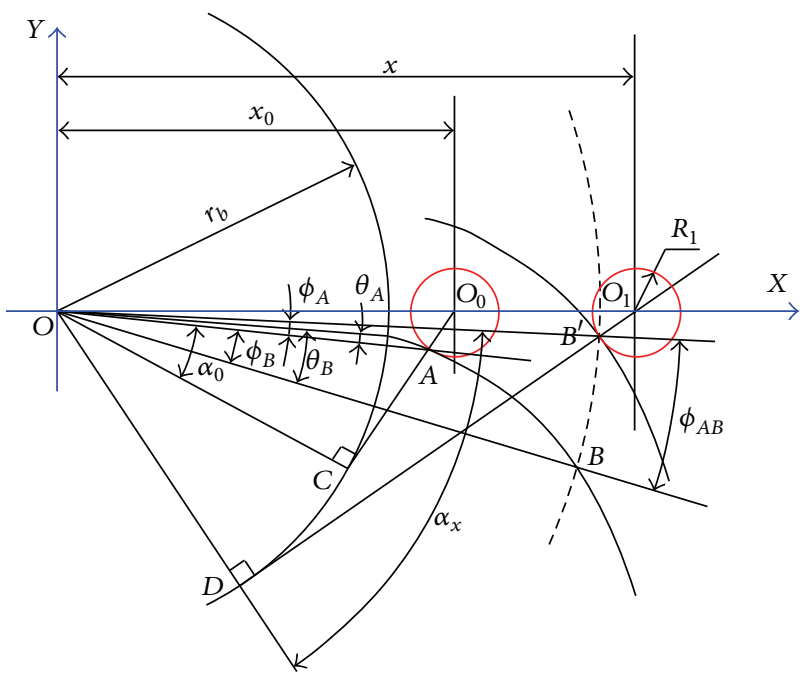

FIGURE 2: Schematic illustration of polar coordinate measurement method.

involute curve is achieved by axes $C$ and $X$ when they are moving at the same time. Because of the motion limit in axial $Y$ direction, polar coordinate measurement is a major method of on-machine measurement when measuring profile error on the CNC gear form grinder. The mathematical model of polar coordinate method measurement is established according to the workpiece rotary movement $(C$ axis) and radial feed movement ( $X$ axis) [17].

On-machine measurement system can not only measure profile error and helical error but also measure pitch error of helical gears. Using the measurement data analysis of these errors, which may correct processing parameters and axial motion equation of polynomial coefficients, realizes the closed-loop feedback processing of tooth surface modification, thus laying the foundation for improving the precision of tooth surface.

The polar coordinates of spherical probe method are used to measure the involute tooth profile as shown in Figure 2; $A$ is the measuring start point; $B$ is the measuring point; $B^{\prime}$ is the actual location of measuring point $B . \phi_{A B}$ is an angle of rotary motion for $C$ axis which can be expressed as

$$
\phi_{A B}=\phi_{A}+\phi_{B}
$$

where

$$
\begin{aligned}
& \phi_{B}=\theta_{B}-\theta_{A}, \\
& \theta_{A}=\tan \alpha_{0}-\alpha_{0}=\frac{\sqrt{x_{0}^{2}-r_{b}^{2}}-R_{1}}{r_{b}}-\alpha_{0}, \\
& \theta_{B}=\tan \alpha_{x}-\alpha_{x}=\frac{\sqrt{x^{2}-r_{b}^{2}}-R_{1}}{r_{b}}-\alpha_{x},
\end{aligned}
$$

where $r_{b}$ is the radius of the base cylinder; variable parameters $x_{0}$ and $x$ are coordinate data of probe centre corresponding to start measuring points $A$ and $B$, respectively; $\alpha_{0}$ and $\alpha_{x}$ are the pressure angle in the cross section for the base circle radius corresponding to points $A$ and $B$ in profile, respectively; $\theta_{A}$ and $\theta_{B}$ are the spread angle of an involute curve of the measured gear profile corresponding to points $A$ and $B$ in profile, respectively. $R_{1}$ is the equivalent radius of the spherical probe and can be expressed as $R_{1}=R \cdot \cos \beta_{b}$, where $R$ is the radius of the spherical probe and $\beta_{b}$ is the helix angle on the base circle.

On the other hand,

$$
\begin{aligned}
\phi_{A} & =\angle A O X-\angle B^{\prime} O X \\
& =\arccos \frac{r_{b}}{x_{0}}-\alpha_{0}-\left(\arccos \frac{r_{b}}{x}-\alpha_{x}\right) .
\end{aligned}
$$

In the above equations, rotary angle $\phi_{A B}$ is expressed as

$$
\phi_{A B}=\frac{\sqrt{x^{2}-r_{b}^{2}}}{r_{b}}-\frac{\sqrt{x_{0}^{2}-r_{b}^{2}}}{r_{b}}-\arccos \frac{r_{b}}{x}+\arccos \frac{r_{b}}{x_{0}} .
$$

Generally speaking, when a gear is measured on grinding machine, using the polar coordinate method, the probe will be set in the radial direction of the workpiece in which it is doing rotary motion along its axis. As soon as it touches the surface, a signal is sent to the gear form grinding machine controller, which stops movements of machine axes such as $C$, $X$, and $Z$. So the control system can acquire the real position of the machine, which is converted into coordinates of the contact point of the probe tip with gear tooth surface. When the probe moves, one of the contacts breaks and a binary signal comes out from the probe head. Then, this binary signal is converted into the grinding machine controller immediately. The controller gets the output signal of the probe and latches actual angle of turntable $\phi_{A B}^{\prime}$. Comparing the achieved angle $\phi_{A B}^{\prime}$ and its theoretical value $\phi_{A B}$, error was calculated for the tooth profile. The deviation of the tooth surface is given by

$$
\Delta \rho=r_{b}\left(\phi_{A B}-\phi_{A B}^{\prime}\right) .
$$

During grinding process, the probe is placed below the wheel so that it cannot be destroyed. When measurement is needed to be done, grinding is stopped automatically and probe is stretched out by the air cylinder. Then, the touch probe is taken at a position which is at such a distance apart from the tooth surface. The probe tip will not touch the workpiece while it moves over the workpiece in $X$ and $Z$ directions. When the probe touches the tooth surface, the controller records the coordinate values of axes $C, X$, and $Z$, immediately.

2.3. Determining of the Probe Centre. Helical gears that transform rotation between parallel axes in opposite directions are in external meshing and are provided with screw tooth surfaces of opposite directions. A helical gear tooth surface is generated by an involute curve that performs a screw motion. Segmented topological modification crowns the tooth surface in profile and longitudinal directions at the same time. Profile segment modification is achieved by modifying the grinding 


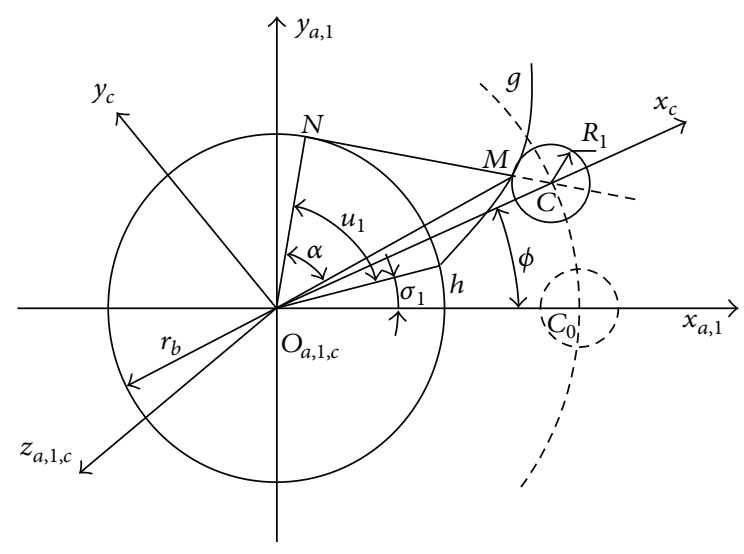

FIGURE 3: Coordinate systems applied to solve the centre of spherical probe coordinates.

wheel, and longitudinal segment modification is completed by changing the grinding path of the wheel relatively to the gear.

As shown in Figure 3, given an involute curve $h g$ in the cross section of tooth surfaces of gear, if you want to measure tooth profile error of any point such as $M$ point, you may set a position in normal direction of the involute curve at point $M$. That is to say, the centre of spherical probe is located in the extension cord of tangent $N M$ on the base circle.

Coordinate systems $S_{1}\left(x_{1}, y_{1}, z_{1}\right)$ and $S_{a}\left(x_{a}, y_{a}, z_{a}\right)$ are rigidly connected to the workpiece and rotary table, respectively. So coordinate system $S_{1}\left(x_{1}, y_{1}, z_{1}\right)$ is named workpiece coordinate system. Coordinate system $S_{c}\left(x_{c}, y_{c}, z_{c}\right)$ is the measurement coordinate system in which the probe rotates the workpiece axis. The coordinate system $S_{c}$ initially coincides with $S_{a}$. Due to no displacement in $Y_{1}$ axial direction as the probe in the measurement, the centre of spherical probe only always can be moved along the $X_{1}$ axial direction.

Any point such as point $M\left(x_{1 M}, y_{1 M}, z_{1 M}\right)$ on the tooth surface measured using on-machine measurement has coordinates, in the workpiece coordinate system $S_{1}\left(x_{1}, y_{1}, z_{1}\right)$, which can be expressed as

$$
\begin{aligned}
& x_{1 M}=r_{b} \cos \left(\sigma_{1}+u_{1}\right)+r_{b} u_{1} \sin \left(\sigma_{1}+u_{1}\right), \\
& y_{1 M}=r_{b} \sin \left(\sigma_{1}+u_{1}\right)-r_{b} u_{1} \cos \left(\sigma_{1}+u_{1}\right), \\
& z_{1 M}=0,
\end{aligned}
$$

where $u_{1}$ is the spread angle of an involute, $\sigma_{1}$ is half of the angular width of the space on the base circle, and $r_{b}$ is the radius of the base cylinder.

Point $C\left(x_{1 C}, y_{1 C}, z_{1 C}\right)$, the centre of spherical probe, may be expressed in coordinate system $S_{1}$ by

$$
\begin{aligned}
& x_{1 C}=x_{1 M}+R \cos \beta_{b} \sin \left(\sigma_{1}+u_{1}\right), \\
& y_{1 C}=y_{1 M}-R \cos \beta_{b} \cos \left(\sigma_{1}+u_{1}\right), \\
& z_{1 C}=R \sin \beta_{b},
\end{aligned}
$$

where $\beta_{b}$ is the helix angle on the base circle.
To keep the centre of spherical probe in the coordinate plane $x_{1} o_{1} z_{1}$, the measurement coordinate system $S_{c}$, the workpiece, and the probe should be together rotated by an angle $\phi$ around the $Z_{1}$ axis. The transformation matrix $S_{c}$ to $S_{1}$ is expressed as follows:

$$
\mathbf{M}_{1 C}=\left[\begin{array}{cccc}
\cos \phi & -\sin \phi & 0 & 0 \\
\sin \phi & \cos \phi & 0 & 0 \\
0 & 0 & 1 & 0 \\
0 & 0 & 0 & 1
\end{array}\right]
$$

For the rotated point $C$, its coordinate point $C\left(x_{1 C 0}, y_{1 C 0}\right.$, $\left.z_{1 C 0}\right)$, the centre of spherical probe, is expressed in coordinate system $S_{1}$ by

$$
\left[\begin{array}{cccc}
\cos \phi & -\sin \phi & 0 & 0 \\
\sin \phi & \cos \phi & 0 & 0 \\
0 & 0 & 1 & 0 \\
0 & 0 & 0 & 1
\end{array}\right]\left[\begin{array}{c}
x_{1 C} \\
y_{1 C} \\
z_{1 C} \\
1
\end{array}\right]=\left[\begin{array}{c}
x_{1 C 0} \\
y_{1 C 0} \\
z_{1 C 0} \\
1
\end{array}\right] .
$$

Because of the centre of spherical probe in the coordinate plane $x_{1} o_{1} z_{1}$ from beginning to end, point $C$ is equal to zero in $Y_{1}$ direction, which can be expressed as

$$
y_{1 C 0}=0 \text {. }
$$

Therefore, comparing (12) with (13) satisfies the following relation:

$$
x_{1 C} \sin \phi+y_{1 C} \cos \phi=0 .
$$

So the rotated angle $\phi$ can be determined as follows:

$$
\begin{aligned}
\phi & =-\arctan \frac{y_{1 C}}{x_{1 C}} \\
& =\arctan \frac{-y_{1 M}+R \cos \beta_{b} \cos \left(\sigma_{1}+u_{1}\right)}{x_{1 M}+R \cos \beta_{b} \sin \left(\sigma_{1}+u_{1}\right)} .
\end{aligned}
$$

Consequently, the coordinates $\left(x_{1 C 0}, y_{1 C 0}\right.$, and $\left.z_{1 C 0}\right)$ can be obtained in coordinate system $S_{1}$. Coordinate system $S_{1}$ is introduced by the tooth surface generation modeling and it is rigidly fixed to the gear with $Z_{1}$ axis coinciding with the axis of the gear. Hence,

$$
\begin{aligned}
x_{1 C 0}= & {\left[x_{1 M}+R \cos \beta_{b} \sin \left(\sigma_{1}+u_{1}\right)\right] \cos \phi } \\
& -\left[y_{1 M}-R \cos \beta_{b} \cos \left(\sigma_{1}+u_{1}\right)\right] \sin \phi, \\
y_{1 C 0}= & 0, \\
z_{1 C 0}= & z_{1 M}+R \sin \beta_{b} .
\end{aligned}
$$

2.4. Generation of Grid Point of Tooth Surface. For measuring theoretical data, the tooth surface equation of measured gear must be known. Assuming the grinding parameters of the gear pair are known, a series of coordinate transformation and derivation processes are needed to get the tooth surface equation. Here, the topological modification is carried out 

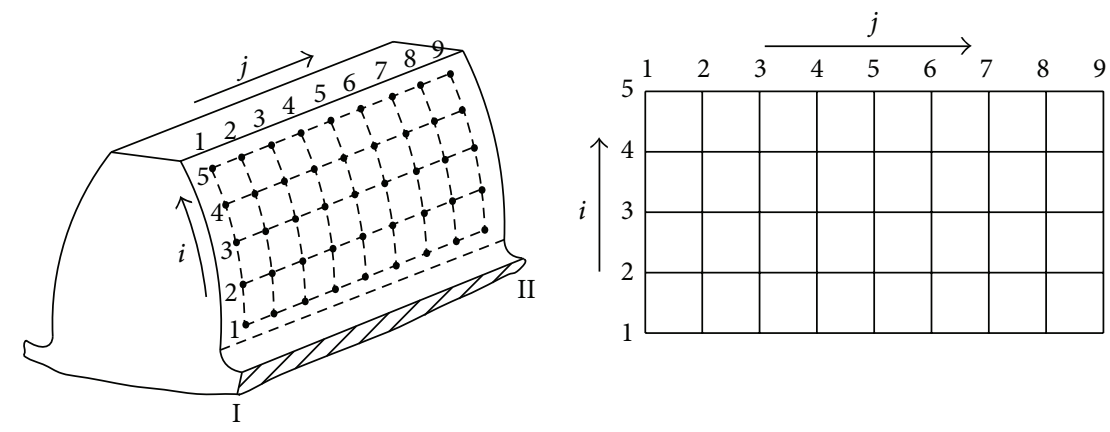

FIGURE 4: Definition of tooth flank grid.

on the driving gear; on the other hand, the driven gear is a standard involute gear. For the topological modification tooth surface, tooth surface measurement areas need to be planned to get the whole tooth surface error information. According to the relevant standards of American gear measurement, the division of tooth surface grid in the longitudinal direction should be less than $10 \%$ of the tooth width; in the profile direction, it should be less than $5 \%$ of the working depth but must be less than or equal to $0.6 \mathrm{~mm}$. The density of tooth surface measurement point is generally taken to be 9 columns long in the longitudinal direction and taken to be 5 rows in the profile direction, a total of 45 points. The tooth surface measurement path planning is shown in Figure 4.

The plane coordinate system $X O Z$ is an axis of rotation projection section of the tooth surface, where $O$ is centre point of base circle and $M^{*}$ is a point in the projection surface at any measured point in the tooth surface. Given a point by $\left(x^{*}, z^{*}\right)$, the following system of nonlinear equations can be used to solve for the surface parameters:

$$
\begin{array}{r}
\sqrt{x_{i}^{2}+y_{i}^{2}}=x^{*} \\
z_{i}=z^{*},
\end{array}
$$

where the coordinates $\left(x_{i}, y_{i}\right.$, and $\left.z_{i}\right)$ are three coordinate components of tooth surface position vector $\mathbf{r}_{1}^{(i)}, i=1,2$, $\ldots, 45$.

The tooth surface of topological modification is no longer the involute tooth surface and becomes complex because of the existence of machining error. So the whole tooth surface needs to be digitally processed to get more accurate tooth surface geometric shape, namely, dividing grid on the tooth surface and computing the coordinate data of the grid point. Figure 5 shows the partition of the grid point inside gear shaft section after rotation projection, in which the grid points are divided into 5 rows by 9 columns.

In Figure $5, A_{1}, A_{2}, A_{3}$, and $A_{4}$ are the four boundary points of the tooth surface; $A_{1}^{\prime}, A_{2}^{\prime}, A_{3}^{\prime}$, and $A_{4}^{\prime}$ are the four new boundary points corresponding to tooth surface contraction grid area. Here, $\Delta l_{1}$ is the top contraction, $\Delta l_{2}$ is the front contraction, $\Delta l_{3}$ is the root contraction, and $\Delta l_{4}$ is the back contraction, respectively. The coordinate of a node $P_{i j}(i=1 \sim 5, j=1 \sim 9)$ in the tooth surface grid is $\left(Z_{i j}, R_{i j}\right)$.

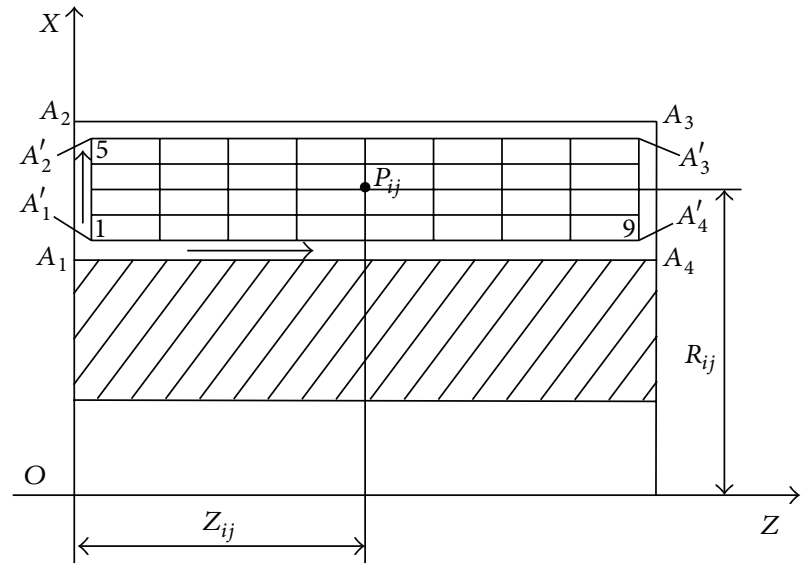

Figure 5: Positions of grid points on one tooth.

The coordinates of the tooth surface boundary points $A_{1}$, $A_{2}, A_{3}$, and $A_{4}$ in the $X O Z$ plane are, respectively, shown as follows:

$$
\begin{aligned}
& A_{1}:\left\{\begin{array}{l}
Z_{A 1}=0 \\
X_{A 1}=r_{f},
\end{array}\right. \\
& A_{2}:\left\{\begin{array}{l}
Z_{A 2}=0 \\
X_{A 2}=r_{a},
\end{array}\right. \\
& A_{3}:\left\{\begin{array}{l}
Z_{A 3}=b \\
X_{A 3}=r_{a},
\end{array}\right. \\
& A_{4}:\left\{\begin{array}{l}
Z_{A 4}=b \\
X_{A 4}=r_{f} ;
\end{array}\right.
\end{aligned}
$$

here, $r_{f}$ is the tooth root radius, $r_{a}$ is the tooth top radius, and $b$ is the tooth width, respectively. 
The new coordinates of tooth surface boundary points $A_{1}^{\prime}, A_{2}^{\prime}, A_{3}^{\prime}$, and $A_{4}^{\prime}$ in the $X O Z$ plane are, respectively, shown as follows:

$$
\begin{aligned}
& A_{1}^{\prime}:\left\{\begin{array}{l}
Z_{A 1}=\Delta l_{4} \\
X_{A 1}=r_{f}+\Delta l_{3},
\end{array}\right. \\
& A_{2}^{\prime}:\left\{\begin{array}{l}
Z_{A 2}=\Delta l_{4} \\
X_{A 2}=r_{a}-\Delta l_{1},
\end{array}\right. \\
& A_{3}^{\prime}:\left\{\begin{array}{l}
Z_{A 3}=b-\Delta l_{2} \\
X_{A 3}=r_{a}-\Delta l_{1},
\end{array}\right. \\
& A_{4}^{\prime}:\left\{\begin{array}{l}
Z_{A 4}=b-\Delta l_{2} \\
X_{A 4}=r_{f}+\Delta l_{3} .
\end{array}\right.
\end{aligned}
$$

The coordinates $\left(Z_{1 j}, X_{1 j}\right)(j=1 \sim 9)$ of 9 equal-division points $P_{1 j}$ between $A_{1}^{\prime}$ and $A_{4}^{\prime}$ in the $X O Z$ plane are shown as follows:

$$
\begin{aligned}
& Z_{1 j}=\Delta l_{4}+\frac{j-1}{8}\left(b-\Delta l_{2}-\Delta l_{4}\right), \\
& X_{1 j}=r_{f}+\Delta l_{3} .
\end{aligned}
$$

The coordinates $\left(Z_{5 j}, X_{5 j}\right)(j=1 \sim 9)$ of 9 equal-division points $P_{5 j}$ between $A_{2}^{\prime}$ and $A_{3}^{\prime}$ in the $X O Z$ plane are shown as follows:

$$
\begin{aligned}
& Z_{5 j}=\Delta l_{4}+\frac{j-1}{8}\left(b-\Delta l_{2}-\Delta l_{4}\right), \\
& X_{5 j}=r_{a}-\Delta l_{1} .
\end{aligned}
$$

The coordinates $\left(Z_{i 1}, X_{i 1}\right)(i=1 \sim 5)$ of 9 equal-division points $P_{i 1}$ between $A_{1}^{\prime}$ and $A_{2}^{\prime}$ in the $X O Z$ plane are shown as follows:

$$
\begin{aligned}
& Z_{i 1}=\Delta l_{4}, \\
& X_{i 1}=r_{f}+\Delta l_{3}+\frac{i-1}{4}\left(r_{a}-r_{f}-\Delta l_{1}-\Delta l_{3}\right) .
\end{aligned}
$$

The coordinates $\left(Z_{i 9}, X_{i 9}\right)(i=1 \sim 5)$ of 9 equal-division points $P_{i 9}$ between $A_{4}^{\prime}$ and $A_{3}^{\prime}$ in the $X O Z$ plane are shown as follows:

$$
\begin{aligned}
& Z_{i 9}=b-\Delta l_{2}, \\
& X_{i 9}=r_{f}+\Delta l_{3}+\frac{i-1}{4}\left(r_{a}-r_{f}-\Delta l_{1}-\Delta l_{3}\right) .
\end{aligned}
$$

From the above derivational process, the coordinates $\left(Z_{i j}, X_{i j}\right)(i=1 \sim 5, j=1 \sim 9)$ of any point $P_{i j}$ in the tooth surface grid in the $X O Z$ plane can be calculated by the formulae that are shown as follows:

$$
\begin{aligned}
& Z_{i j}=\Delta l_{4}+\frac{j-1}{8}\left(b-\Delta l_{2}-\Delta l_{4}\right), \\
& X_{i j}=r_{f}+\Delta l_{3}+\frac{i-1}{4}\left(r_{a}-r_{f}-\Delta l_{1}-\Delta l_{3}\right) .
\end{aligned}
$$

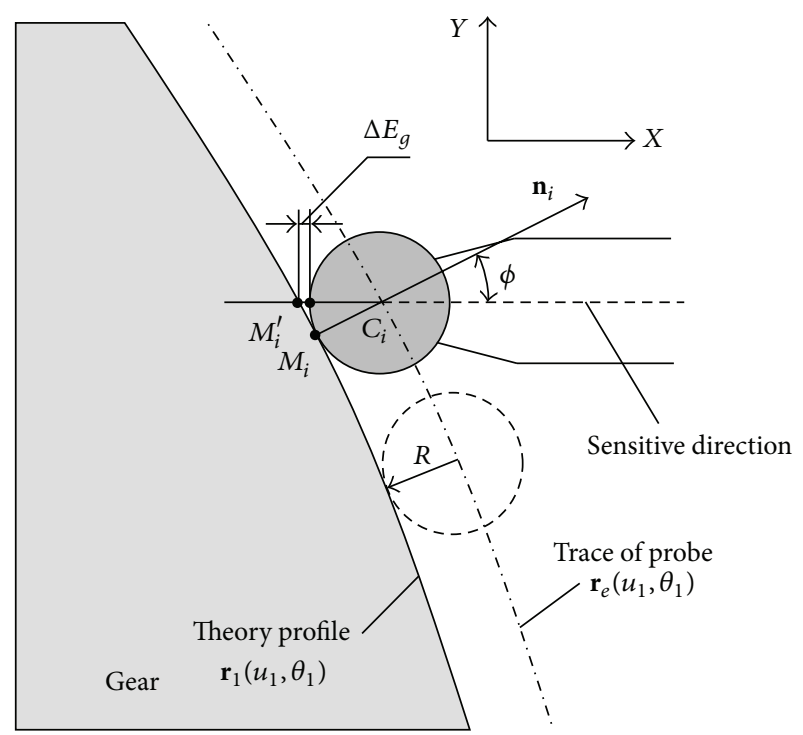

FIGURE 6: Relationships of the probe geometry and theory profile.

Solving nonlinear equations to get the tooth surface parameters $\left(u_{1}, \theta_{1}\right)$ for each measured point, which substitute into (2), can get the theoretical coordinates $\left(x_{1}^{(i)}, y_{1}^{(i)}\right.$, and $\left.z_{1}^{(i)}\right)$ and the unit normal vector $\mathbf{n}_{i}$. Hence,

$$
\begin{aligned}
\mathbf{r}_{1}^{(i)} & =\left[\begin{array}{lll}
x_{1}^{(i)} & y_{1}^{(i)} & z_{1}^{(i)}
\end{array}\right]^{T}, \\
\mathbf{n}_{1}^{(i)} & =\left[\begin{array}{lll}
n_{1 x}^{(i)} & n_{1 y}^{(i)} & n_{1 z}^{(i)}
\end{array}\right]^{T} \\
& (i=1,2, \ldots, 2 \times 45) .
\end{aligned}
$$

2.5. Measurement Error Compensation. When a point is measured on the modification gear tooth surface, the inspection datum of an on-machine measurement is the centre of the probe. To improve the measurement accuracy, the probe radius can be considered in calculating the profile errors. Figure 6 schematically shows the effect of the probe size on the measurement. As the probe always has a certain dimension, an error $\Delta E_{g}$ is unavoidable. Due to the size effect of the probe, the actual contact point of the probe is at point $M_{i}$, instead of $M_{i}^{\prime}$.

According to the envelope surface characteristics, the normal vector is perpendicular to the probe sphere and passed through the centre of the probe $C_{i}$. The theoretical motion trajectory of probe centre is shown as follows:

$$
\mathbf{r}_{e}\left(u_{1}, \theta_{1}\right)=\mathbf{r}_{1}\left(u_{1}, \theta_{1}\right)+R \cdot \mathbf{n}_{1}\left(u_{1}, \theta_{1}\right) ;
$$

here, $\mathbf{r}_{e}\left(u_{1}, \theta_{1}\right)$ is the trace of the probe centre and $R$ is the probe radius.

Strictly speaking, the measuring tooth surface deviation is the deviation of the actual contact point $M_{i}$ in the sensitive direction of the probe, so an important factor of the measurement error is the actual contacting position of the probe. In the measurement, the probe is also very close to the contact point; microscopically, the nearness of the contact point $M_{i}$ can approximately be regarded as a small plane. The 
TABLE 1: Parameters of the gears in the trials.

\begin{tabular}{lccc}
\hline Items & Symbol & Unit & Data \\
\hline Driving gear & $z_{1}$ & - & 30 \\
Driven gear & $z_{2}$ & - & 30 \\
Normal model & $m_{n}$ & $\mathrm{~mm}$ & 6.5 \\
Pressure angle & $\alpha_{n}$ & $\mathrm{deg}$ & 20 \\
Helix angle & $\beta$ & $\mathrm{deg}$ & 13 \\
Face width & $b$ & $\mathrm{~mm}$ & 53 \\
Top profile crowning & $a_{m p(c f)}$ & $1 / \mathrm{mm}$ & 0.0011 \\
Bottom profile crowning & $a_{m p(d e)}$ & $1 / \mathrm{mm}$ & 0.0014 \\
Top limit angle & $u_{c}$ & $\mathrm{rad}$ & 0.496 \\
Bottom limit angle & $u_{d}$ & $\mathrm{rad}$ & 0.196 \\
Front longitudinal crowning & $a_{m l(h a)}$ & $1 / \mathrm{mm}$ & 0.0012 \\
Back longitudinal crowning & $a_{m l(b j)}$ & $1 / \mathrm{mm}$ & 0.0012 \\
Front limit angle & $\theta_{a}$ & $\mathrm{rad}$ & 0.018 \\
Back limit angle & $\theta_{b}$ & $\mathrm{rad}$ & 0.104 \\
\hline
\end{tabular}

measurement error caused by probe radius is $\Delta E_{g}$, and its geometric relationships with probe radius can be expressed as follows:

$$
\Delta E_{g}=R\left(\frac{1}{\cos \phi}-1\right) .
$$

According to (27), the larger the probe radius $R$, the greater the measurement error, so the probe radius should be small; here the radius $R$ is $2 \mathrm{~mm}$. The normal direction of the measuring points and the sensitivity of the probe are also included in the XOY plane, and experimental results show that the angle $\phi$ is very small.

From Figure 6, it can be seen that the measured head should be in contact with the theoretical point $M_{i}^{\prime}$, and the $X, Y$ direction of the coordinates of the value of a certain amount of compensation can be guaranteed. Assuming that $M_{i}$ coordinates are $\left(x_{i}, y_{i}\right), M_{i}^{\prime}$ coordinates are $\left(x_{i}^{\prime}, y_{i}^{\prime}\right)$, so the relationship between the two expressions is

$$
\begin{aligned}
& x_{i}^{\prime}=x_{i}-R \operatorname{tg} \phi, \\
& y_{i}^{\prime}=x_{i}+R \operatorname{tg} \phi \sin \phi .
\end{aligned}
$$

The contact points are in agreement with the theoretical contact points after compensation. The contact position of the measuring head does not affect the measurement results.

\section{Numerical Example}

The parameters of the gears are listed in Table 1. The driving gear is modified in the profile and longitudinal directions at the same time. On the contrary, the tooth surface of the driven gear is a conventional screw involute surface. Then, coordinates on the tooth surface are measured and calculated using a MATLAB code.

The measurement data of tooth surface can be obtained by measuring the grid points in the order with the probe. Selecting the radius of the probe $R=2 \mathrm{~mm}$, the probe centre trajectory curve is the envelope surface of measured

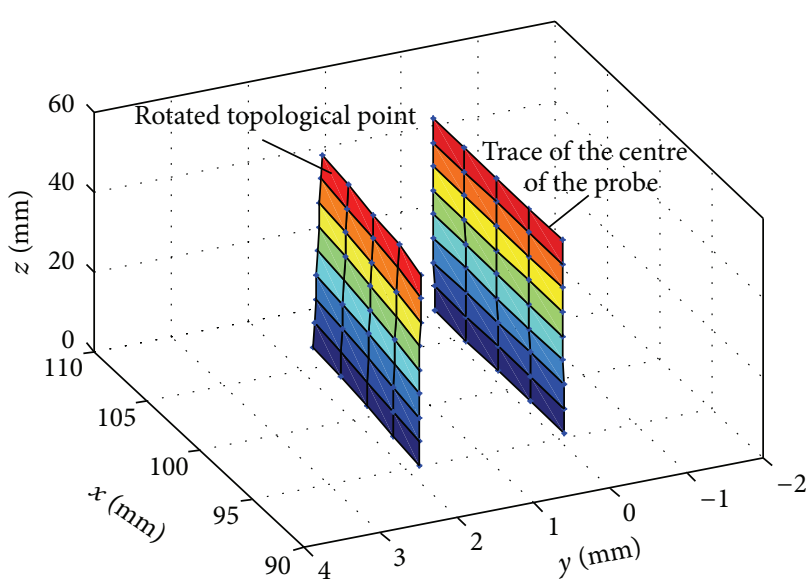

FIgURE 7: The contrast position of rotated topological point and the probe centre.

tooth surface. When the tooth surface is detected using polar coordinate method, the trajectory coordinates of the probe centre and the gear rotating angle $\phi$ are calculated using the control unit. The relationship of relative position between the topology points in the rotated tooth surface and the trajectory of probe centre is shown in Figure 7.

\section{Tooth Flank Errors Separation}

According to the predetermined spacing, the tooth surface deviation of each measured tooth surface detection point is point-to-point measured by probe along the profile and longitudinal directions of the gear. $\mathbf{r}_{0}\left(u_{1}, \theta_{1}\right)$ is the standard involute tooth surface vector, $\mathbf{r}_{1}\left(u_{1}, \theta_{1}\right)$ is the theoretical topological modification tooth surface vector, and $\mathbf{r}_{1 s}\left(u_{1}, \theta_{1}\right)$ is the actual tooth surface vector after grinding, respectively. Due to the error of the machine tool adjustment and movement, the actual tooth surface often deviates from the theoretical tooth surface. The tooth surface error $\Delta E$ is usually measured along the direction of the unit normal vector $\mathbf{n}_{1}$ and is presented as

$$
\Delta E=\left(\mathbf{r}_{1 s}\left(u_{1}, \theta_{1}\right)-\mathbf{r}_{1}\left(u_{1}, \theta_{1}\right)\right) \cdot \mathbf{n}_{1}\left(u_{1}, \theta_{1}\right) .
$$

According to the standard involute tooth surface vector $\mathbf{r}_{0}\left(u_{1}, \theta_{1}\right)$ and the modified tooth surface vector $\mathbf{r}_{1}\left(u_{1}, \theta_{1}\right)$, the topological modification amount $\delta$ can be obtained which is shown as follows:

$$
\delta=\left(\mathbf{r}_{1}\left(u_{1}, \theta_{1}\right)-\mathbf{r}_{0}\left(u_{1}, \theta_{1}\right)\right) \cdot \mathbf{n}_{0}\left(u_{1}, \theta_{1}\right) .
$$

If the tooth surface topology deviation is expressed as $\Delta \rho$, the tooth surface error $\Delta E$ can be expressed by $\Delta \rho$ and $\delta$ as follows:

$$
\Delta E=\Delta \rho-\delta .
$$

Equation (31) shows that the tooth surface error can be obtained by removing the modification amount out of the topology deviation of tooth surface. 
TABLE 2: The modification amount and tooth surface error data (unit: $\mathrm{mm}$ ).

\begin{tabular}{lccccccccccc}
\hline Location & & 1 & 2 & 3 & 4 & 5 & 6 & 7 & 8 & 9 \\
\hline & 1 & -0.0564 & -0.0436 & -0.0314 & -0.0257 & -0.0239 & -0.0285 & -0.0291 & -0.0385 & -0.0497 \\
& 2 & -0.0449 & -0.0387 & -0.0215 & -0.0159 & -0.0145 & -0.0147 & -0.0214 & -0.0289 & -0.0436 \\
Right flank/mm & 3 & -0.0406 & -0.0302 & -0.0197 & -0.0115 & -0.0027 & -0.012 & -0.0173 & -0.0237 & -0.0391 \\
& 4 & -0.0479 & -0.0421 & -0.0236 & -0.0214 & -0.0136 & -0.0234 & -0.0257 & -0.0356 & -0.0412 \\
& 5 & -0.0535 & -0.0509 & -0.0352 & -0.0298 & -0.0172 & -0.0287 & -0.0327 & -0.0492 & -0.0518 \\
\hline & & 1 & 2 & 3 & 4 & 5 & 6 & 7 & 8 & 9 \\
Left flank/mm & 5 & -0.0524 & -0.0487 & -0.0301 & -0.0272 & -0.0196 & -0.0264 & -0.0314 & -0.0501 & -0.0544 \\
& 4 & -0.0498 & -0.0410 & -0.0198 & -0.0189 & -0.0035 & -0.0197 & -0.0241 & -0.0417 & -0.0463 \\
& 3 & -0.0436 & -0.0375 & -0.0221 & -0.0035 & -0.0021 & -0.0046 & -0.0210 & -0.0324 & -0.0452 \\
& 2 & -0.0510 & -0.0417 & -0.0183 & -0.0167 & -0.0138 & -0.0153 & -0.0189 & -0.0394 & -0.0531 \\
& 1 & -0.0571 & -0.0462 & -0.0294 & -0.0285 & -0.0227 & -0.0249 & -0.0267 & -0.0472 & -0.0592 \\
\hline
\end{tabular}

TABLE 3: The tooth surface error data (unit: $\mathrm{mm}$ ).

\begin{tabular}{lccccccccccc}
\hline Location & & 1 & 2 & 3 & 4 & 5 & 6 & 7 & 8 & 9 \\
\hline & 1 & -0.0044 & -0.0047 & -0.0046 & -0.0046 & -0.0062 & -0.0062 & -0.0066 & -0.0061 & -0.0061 \\
& 2 & -0.0061 & -0.0064 & -0.0063 & -0.0064 & -0.0079 & -0.0079 & -0.0083 & -0.0077 & -0.0079 \\
Right flank/mm & 3 & -0.0058 & -0.0061 & -0.0062 & -0.0063 & -0.0075 & -0.0076 & -0.0084 & -0.0074 & -0.0075 \\
& 4 & -0.0056 & -0.0059 & -0.0058 & -0.0059 & -0.0074 & -0.0074 & -0.0078 & -0.0072 & -0.0074 \\
& 5 & -0.0048 & -0.0051 & -0.0050 & -0.0051 & -0.0066 & -0.0066 & -0.0071 & -0.0064 & -0.0066 \\
\hline & & 1 & 2 & 3 & 4 & 5 & 6 & 7 & 8 & 9 \\
Left flank/mm & 5 & -0.0053 & -0.0057 & -0.0055 & -0.0056 & -0.0057 & -0.0058 & -0.0087 & -0.0054 & -0.0065 \\
& 4 & -0.0062 & -0.0062 & -0.0068 & -0.0072 & -0.0084 & -0.0067 & -0.0086 & -0.0039 & -0.0074 \\
& 3 & -0.0049 & -0.0074 & -0.0072 & -0.0058 & -0.0054 & -0.0069 & -0.0094 & -0.0075 & -0.0087 \\
& 2 & -0.0072 & -0.0049 & -0.0085 & -0.0063 & -0.0072 & -0.0069 & -0.0064 & -0.0058 & -0.0076 \\
& 1 & -0.0088 & -0.0076 & -0.0067 & -0.0053 & -0.0069 & -0.0072 & -0.0073 & -0.0067 & -0.0069 \\
\hline
\end{tabular}

The tooth surface topology deviation $\Delta \rho$ is shown in Table 2 and the tooth surface error $\Delta E$ is shown in Table 3, respectively.

The deviation distribution (error and modification amount) of the tooth surface obtained by an on-machine measurement is shown in Figure 8. The dashed parts (the superposition of error and modification) in regions 2, 4, 6 , and 8 of the tooth surface are obviously presented as a parabolic shape in the profile and longitudinal directions, respectively. The maximum value of deviation at the tooth top is $-0.0571 \mathrm{~mm}$ and the error in the centre of tooth surface area 1 is $-0.0049 \mathrm{~mm}$. The trend is gradually increased towards both sides presenting the shape where the middle is high and both sides are low.

The measurement using the topological modification tooth surface equation is shown in Figure 9. The measured result reflects the actual tooth surface error, the distribution of which has unobvious trend. The maximum error in the tooth top is $-0.0088 \mathrm{~mm}$ and the error in the centre of right tooth surface area 1 is $-0.0054 \mathrm{~mm}$.

To separate flank form deviations from modification amount and to improve the perception of deviation diagrams, all flank deviations are referenced by the modification amount. The characterization of the tooth surface error and modification amount are shown in Figures 10 and 11. The maximum error is about $-8.8 \mathrm{um}$ in the upper and lower

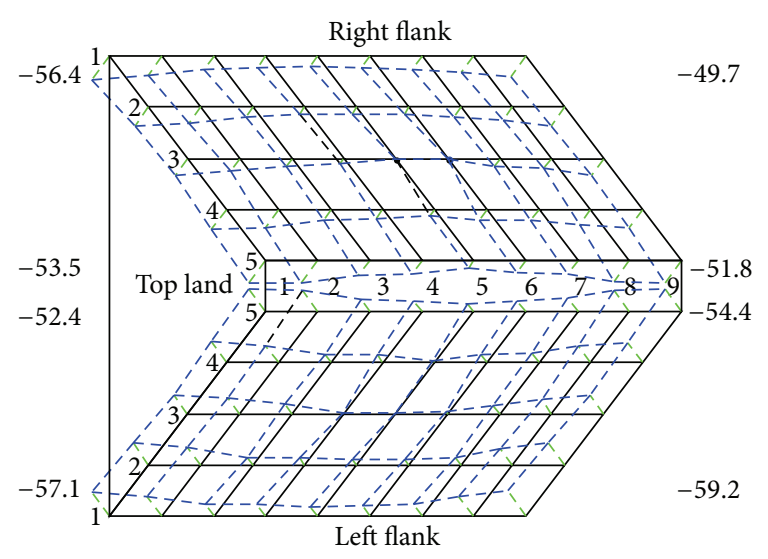

FIGURE 8: Topography diagram of modification amount and error (unit: um).

surface, but the minimum error is about -5.4 um which is concentrated in the central area of the profile and longitudinal directions. The normal error of any intersection of profile direction and longitudinal direction is obtained by the error of the contour map. 


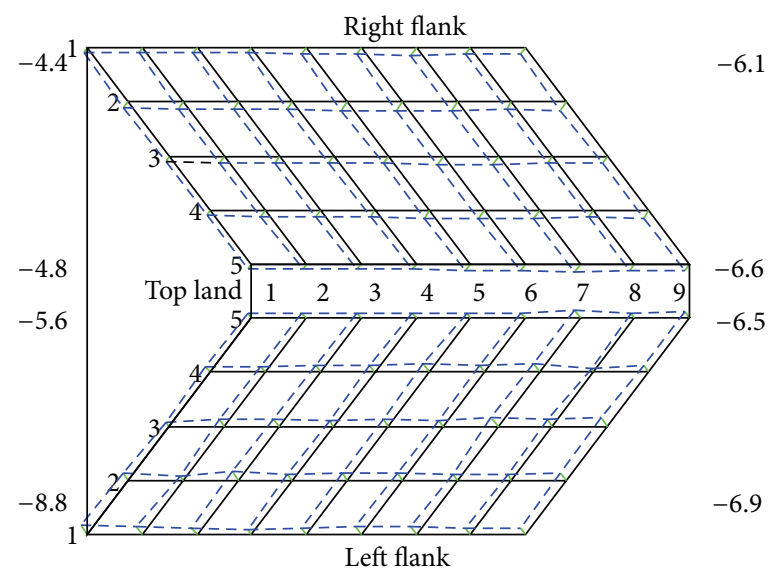

FIgURE 9: Topography diagram of error (unit: um).

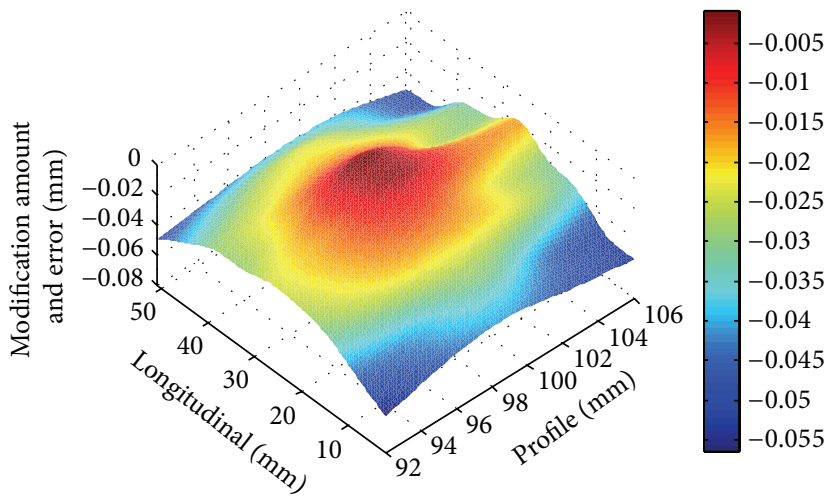

(a) Right flank

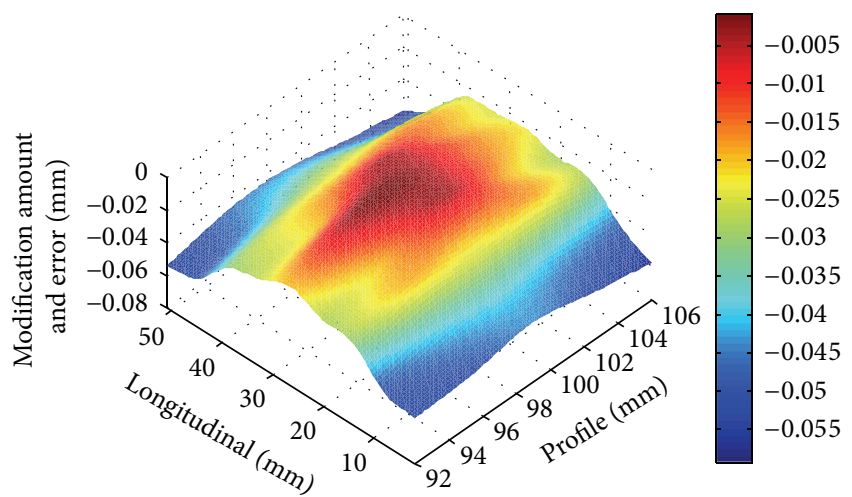

(b) Left flank

FIGURE 10: The contour map of modification amount and error (unit: um).

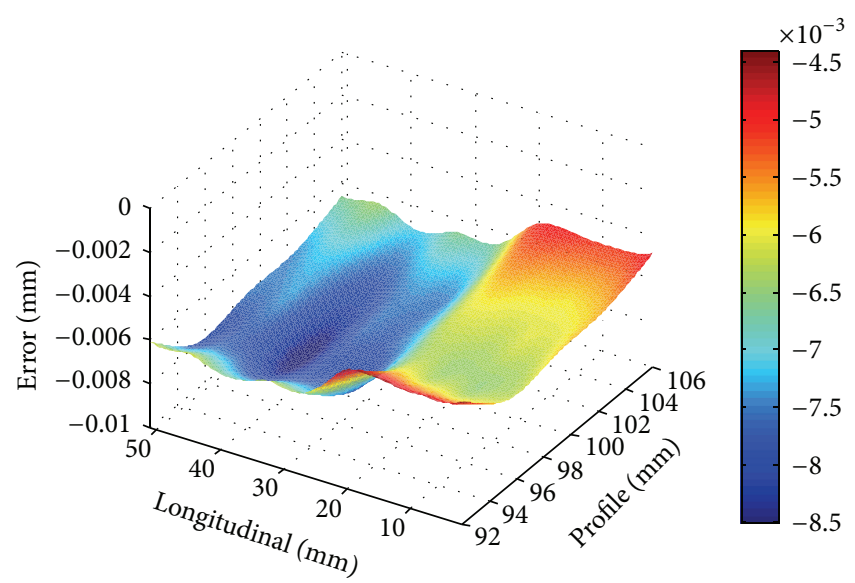

(a) Right flank

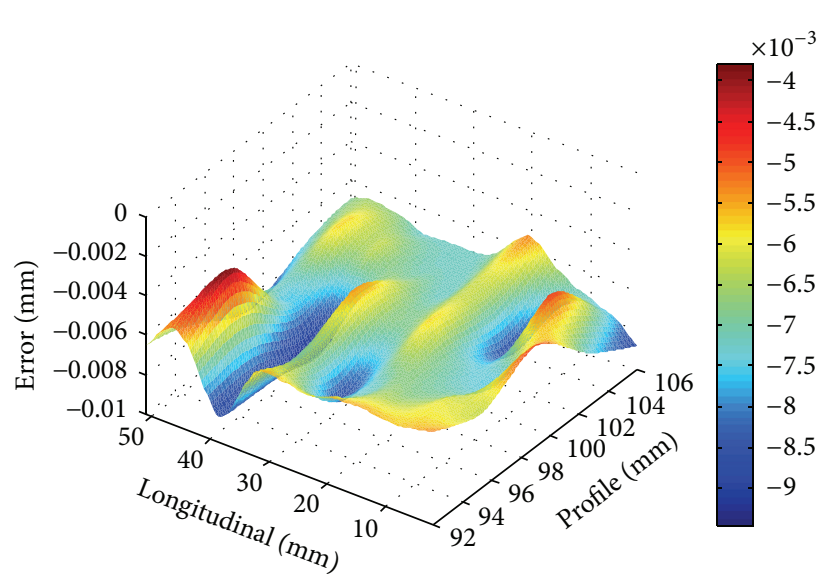

(b) Left flank

FIGURE 11: The contour map of error (unit: um).

\section{Conclusions}

In this study, an on-machine profile measurement system along with a five-axis CNC gear form grinding machine is developed to improve accuracy of grinding. This system includes the following steps: (1) calculate trajectory of the centre of spherical probe; (2) define gear flanks by a grid of points; (3) obtain the coordinate values of topology measurement points. Grinding experiments are performed to verify the accuracy and efficiency of the topography measurements. 
With contour map, the profile and longitudinal directions of the error changes are easily seen. Using statistical process control techniques to monitor the grinding process, can timely attain the detection of changes in the product error exceptions and take the necessary measures to prevent the occurrence of waste. As the numerical examples show, onmachine measurement method can inspect tooth quality. An additional advantage of the approach is to characterize modification amount and tooth surface error, respectively.

\section{Conflict of Interests}

The authors declare that there is no conflict of interests regarding the publication of this paper.

\section{Acknowledgments}

The authors are grateful to the National Natural Science Foundation of China for its financial support. Part of this work was performed under Contracts no. 51375144 and no. 51405135 and the Priority Project of Research in Universities in Henan Province (Grant no. 15A460021).

\section{References}

[1] H. L. Wang, X. Z. Deng, and J. J. Yang, "Form grinding and experiment on segment topographic modification gear," Journal of Aerospace Power, vol. 29, no. 12, pp. 3000-3008, 2014.

[2] H.-Y. You, P.-Q. Ye, J.-S. Wang, and X.-Y. Deng, "Design and application of CBN shape grinding wheel for gears," International Journal of Machine Tools and Manufacture, vol. 43, no. 12, pp. 1269-1277, 2003.

[3] Q. Fan, R. S. Dafoe, and J. W. Swanger, "Higher-order tooth flank form error correction for face-milled spiral bevel and hypoid gears," Journal of Mechanical Design-Transactions of the ASME, vol. 130, no. 7, Article ID 072601, 2008.

[4] J. Argyris, M. De Donno, and F. L. Litvin, "Computer program in visual basic language for simulation of meshing and contact of gear drives and its application for design of worm gear drive," Computer Methods in Applied Mechanics and Engineering, vol. 189, no. 2, pp. 595-612, 2000.

[5] Z. L. Zhang, Y. Fu, Q. R. Yin, and Y. Zeng, "Study on the polar method of measuring of gear profile errors," Chinese Journal of Mechanical Engineering, vol. 37, no. 4, pp. 70-72, 2001.

[6] C. H. Gao, K. Cheng, and D. Webb, "Investigation on sampling size optimisation in gear tooth surface measurement using a CMM," The International Journal of Advanced Manufacturing Technology, vol. 24, no. 7-8, pp. 599-606, 2004.

[7] Z. L. Zhang, Y. Fu, and Y. Zeng, "Expressing gear involute error by polar angle \& generating angle in polar coordinate measuring method," Tool Engineering, vol. 34, no. 4, pp. 39-40, 2000.

[8] F. Gao, B. H. Zhao, and Y. Li, "Novel pre-travel calibration method of touch trigger probe based on error separation," Chinese Journal of Scientific Instrument, vol. 34, no. 7, pp. 15811587, 2013.

[9] F. Gao, Y. Li, S. Tian, Y. Huang, L. Hao, and J. Wang, "Study on the on-machine measurement method of NC wheel gear form grinding machine," Chinese Journal of Scientific Instrument, vol. 29, no. 3, pp. 540-544, 2008.
[10] A. Nafi, J. R. R. Mayer, and A. Wozniak, "Novel CMM-based implementation of the multi-step method for the separation of machine and probe errors," Precision Engineering, vol. 35, no. 2, pp. 318-328, 2011.

[11] A. Nafi, J. R. R. Mayer, and A. Wozniak, "Reduced configuration set for the multi-step method applied to machine and probe error separation on a CMM," Measurement, vol. 45, no. 10, pp. 2321-2329, 2012.

[12] Y.-P. Shih and S.-D. Chen, "Free-form flank correction in helical gear grinding using a five-axis computer numerical control gear profile grinding machine," Journal of Manufacturing Science and Engineering, vol. 134, no. 4, Article ID 041006, 2012.

[13] Y. Kobayashi, N. Nishida, Y. Ougiya, and H. Nagata, “Tooth trace modification processing of helix gear by form grinding method," Transactions of the Japan Society of Mechanical Engineers, Part C, vol. 61, no. 590, pp. 4088-4093, 1995.

[14] Y. Kobayashi, N. Nishida, and Y. Ougiya, "Estimation of grinding wheel setting error in helical gear processing by form grinding," Transactions of the Japan Society of Mechanical Engineers Part C, vol. 63, no. 612, pp. 2852-2858, 1997.

[15] C.-K. Lee, "Manufacturing process for a cylindrical crown gear drive with a controllable fourth order polynomial function of transmission error," Journal of Materials Processing Technology, vol. 209, no. 1, pp. 3-13, 2009.

[16] C. K. Lee and C. K. Chen, "Mathematical models, meshing analysis and transmission design for robust cylindrical gear set generated by double blade-disks with parabolic cutting edges," Proceedings of the Institution of Mechanical Engineers, Part C: Journal of Mechanical Engineering Science, vol. 218, no. 12, pp. 1539-1553, 2004.

[17] Z. Y. Shi and Y. Ye, "Research on the generalized polarcoordinate method for measuring involute profile deviations," Chinese Journal of Scientific Instrument, vol. 22, no. 2, pp. 140$142,2001$. 


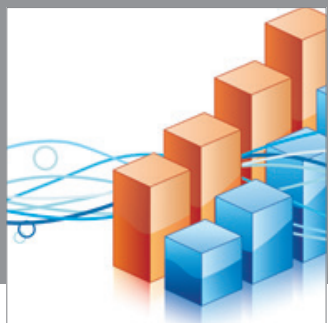

Advances in

Operations Research

mansans

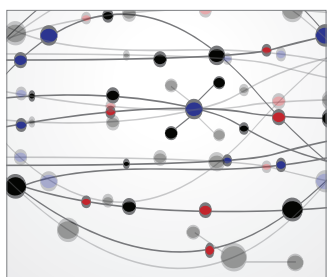

The Scientific World Journal
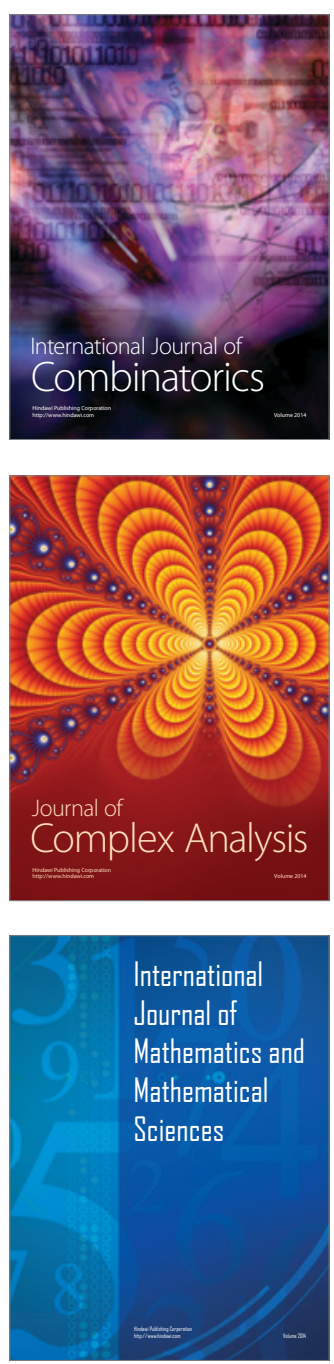
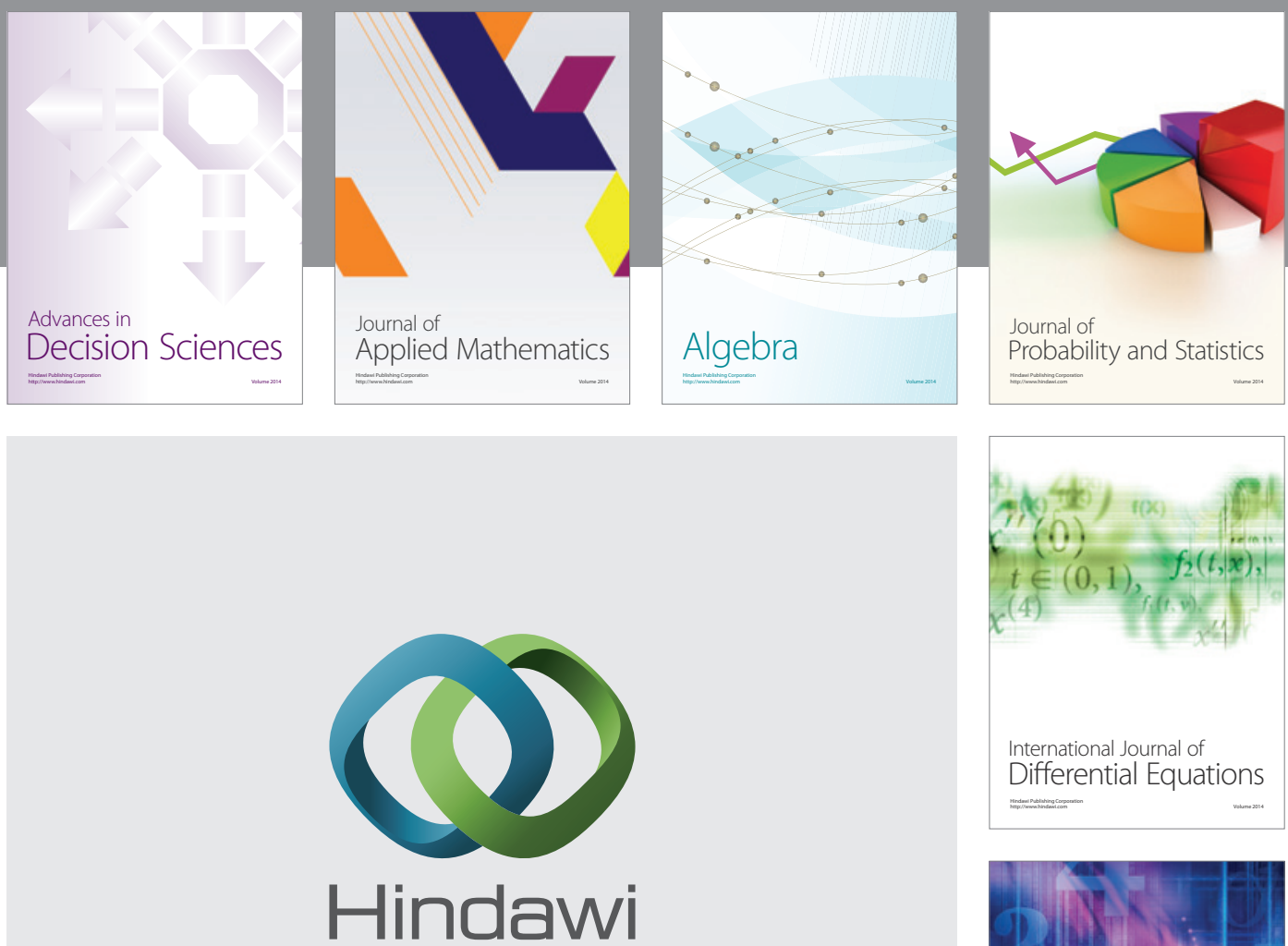

Submit your manuscripts at http://www.hindawi.com
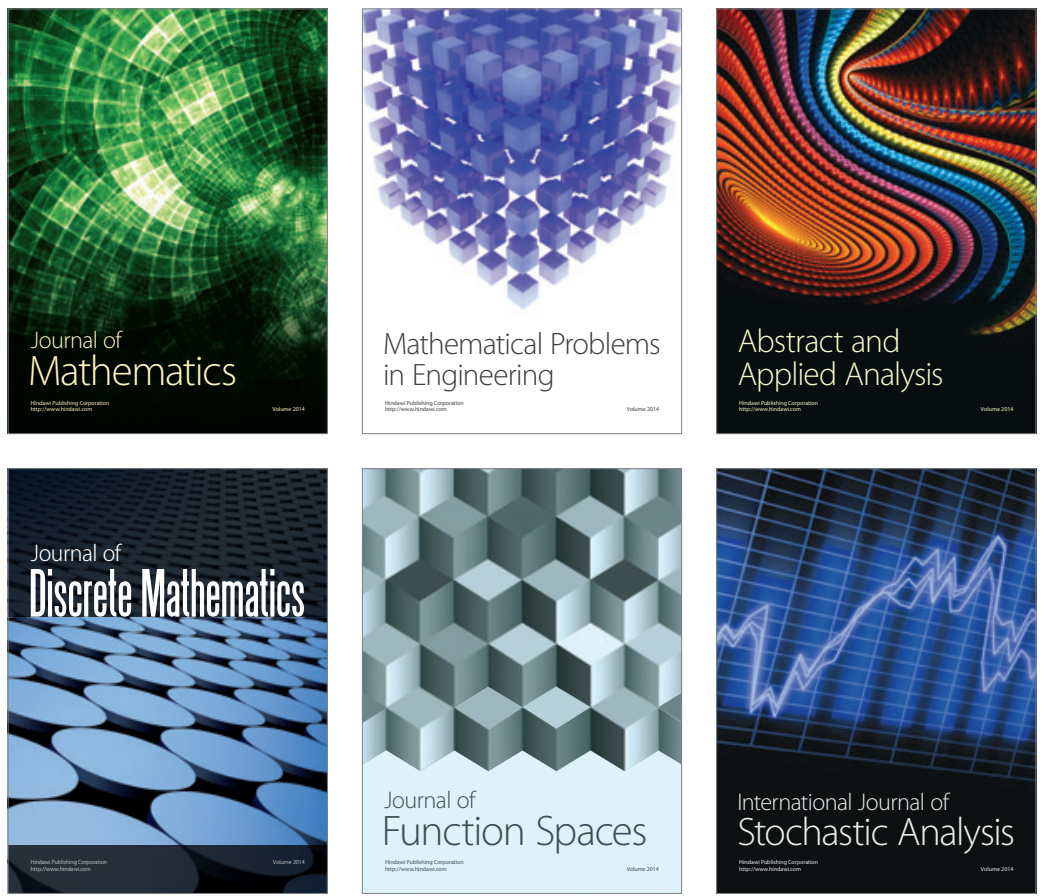

Journal of

Function Spaces

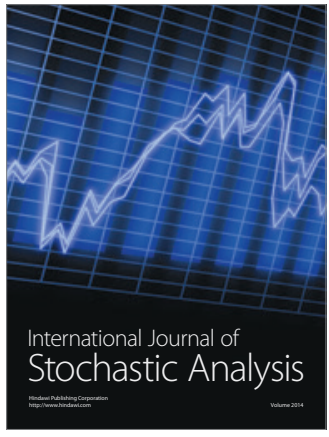

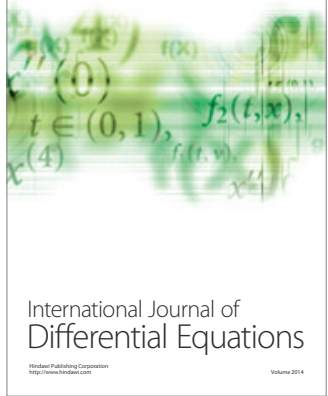
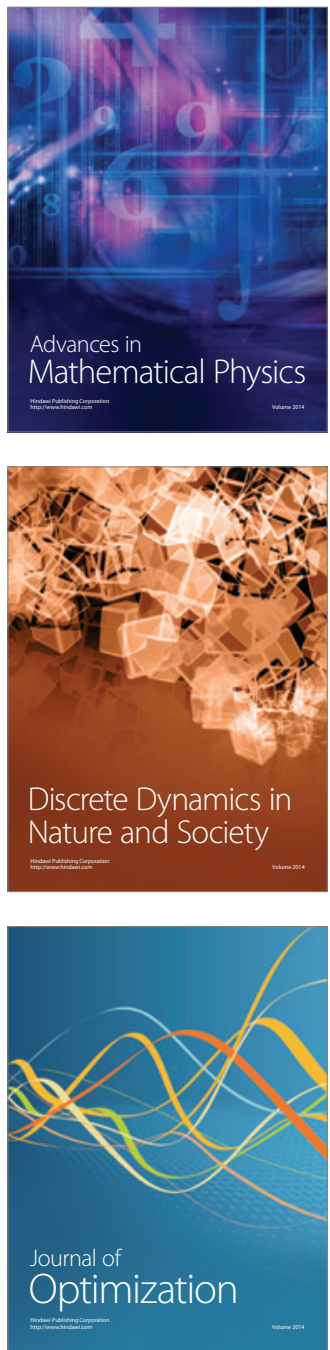